\title{
Internet of Things Technology Based Agricultural Spraying Drone Design for Remote Farming Applications
}

\author{
Cemil Altın ${ }^{1 *}$ (D), Hasan Ulutaş ${ }^{1}$ (ID), Eyyüp $\operatorname{Orhan}^{1}$ (D), $\operatorname{Orhan}^{\text {Er }}{ }^{1}$ (D), Volkan Akdoğan ${ }^{1}$ (D) \\ ${ }^{1}$ Yozgat Bozok University \\ *cemil.altin@bozok.edu.tr \\ *Orcid: 0000-0001-8892-2795
}

Received: 16 August 2020

Accepted: 15 September 2021

DOI: $10.18466 /$ cbayarfbe. 781368

\begin{abstract}
Internet of things and Drones are two new promising innovative technologies that are inevitable in the internet era. These technologies provide modern solutions for many fields. One of these fields is agriculture. Agriculture plays a pivot role for humankind because more than half of the World's population depends on agriculture. In this study internet of things, technology is applied to a drone that is capable of doing agricultural works like spraying, carrying, and real-time monitoring. An onboard android device that is mounted on the drone is used to manage the drone over the internet by a graphical user interface software designed within the study. The farmer communicates with onboard android device over the internet by remote desktop application to manage drone and get data. The drone will help farmers by getting live data from the farm and do necessary works remotely. This study aims to enable farmers to do remote farming. Agricultural activities have declined in recent years with the increase in migration from the village to the city. Thus, farmers will be able to make remote farming.
\end{abstract}

Keywords: IoT, Precise Farming, Remote Farming, Smart Farming, UAV

\section{Introduction}

The future of the smart World will be based on the Internet of Things (IoT). IoT is a vision about everyday systems and devices being connected to the internet[1]. This facilitates tracking, monitoring, data collection, analysis, and control. Another technology that is popular today is unmanned aerial vehicles (UAV) or drones. Drones are unmanned flying aircraft. The fact that drones have some advantages over other aircraft has brought drones forward. Drone's improved mobility enabled them to be used in every field. Today, drones are used in the military, construction, security, energy, agriculture, entertainment, etc. After referring to the IoT and drones that are up to date in the technology, it is necessary to conduct studies on the usability of these two technological products to facilitate and help human life. In other words in which sectors these two products can be used should be studied. To begin with, it is useful to take a look at the sectors that people are dealing with the most. According to the international labor organization, the largest share of the world population (29.6 percent) is engaged in agriculture, forestry, hunting, and fishing. In other words, it can be said that the number of agricultural workers is higher than in other sectors.

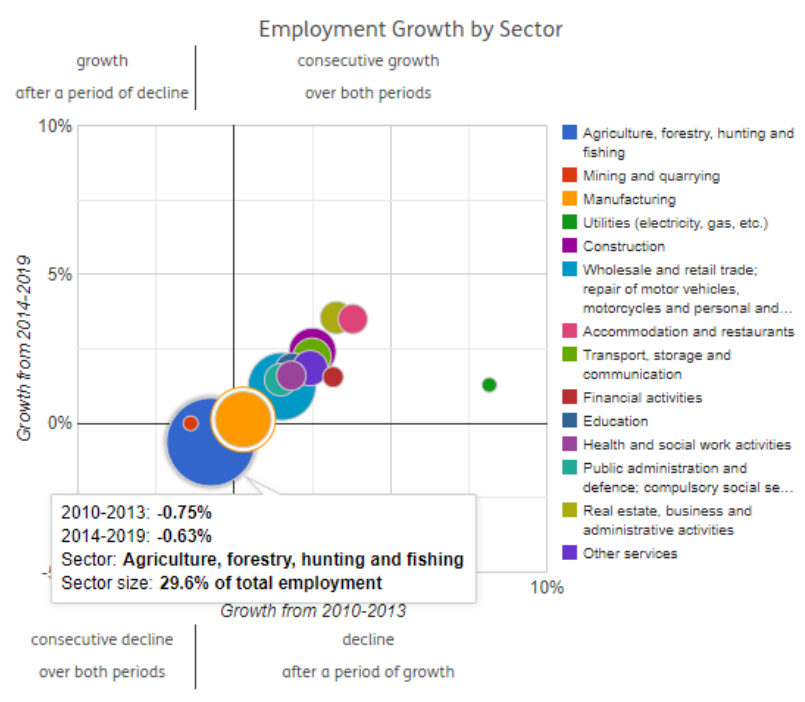

Figure 1. World's employment populations[2].

Figure 1 shows that agriculture has the biggest share. For this reason, the agriculture sector was chosen for the application.

After selecting the sector, it is necessary to identify existing problems in the relevant sector. The problem is 
that agriculture decreases significantly as industrialization increases. As seen in the international labor organization report in Figure 1, the rate of those employed in agriculture has decreased continuously in the period between 2010-2013 (-0.75) and 2014-2019 ($0.63)$. The human population is increasing day by day and thus the need for food increases [3]. Food is a must for the human being to survive.

In recent studies, it is predicted that the world population and food demand will increase rapidly until 2050 [4,5,6]. Studies support each other that food production should increase significantly by 2050 . Therefore, it is important to maintain and increase agricultural production. By using the technology in agriculture, it can easily find a solution to the increasing food demand.

Almost all drones are managed by short-range remote controllers. Due to the short-range problem, drones can only be controlled when they are within view. However, remote farming cannot be done with visual line of sight (VLOS) drones. For remote farming, beyond visual line of sight (BVLOS) drones are needed. Thus, the drone can be controlled even from a very long distance. BVLOS drones are usually drones that can be controlled via the internet. Currently, both agricultural drones and internet-controlled drones are available commercially.

The term agricultural drone is a very comprehensive term. Because there are drones used in a wide variety of agricultural work. Agricultural drones in the literature search; it has been used in jobs such as seed sowing [7], pest detection[8], data acquisition[9], irrigation management[10], plant health monitoring[11,12,13], yield estimation[14], soil analysis[15] and spraying[16,17,18]. Although there are many spraying drones in the literature, IOT-based spraying drones have not been encountered. Existing drones are either spraying drones that can be managed by short-range remote controllers, or IOT based drones especially used for crop monitoring or data acquisition. For this reason, an IOT based spraying drone was designed to be used in spraying jobs in this study.

Multirotor drones are the most suitable unmanned aerial vehicle structure for spraying due to their ability to hang in the air, move slowly and push the drug towards the plant with the help of propellers. For this reason, a multirotor drone was used in the study.

In this paper, a product that is pioneered the use of technology in agriculture and which is important and necessary in the field of agriculture was developed. The main objective of this study is the design of a spraying drone that allows the use of technology in agriculture with affordable price and superior features. Since the designed drone has direct IoT technology, it provides superiority to drones managed over the internet via remote control. Its high carrying capacity of 20 liters is higher than the carrying capacity of many drones.

\section{Materials and Methods \\ 2.1. Chassis Design}

Spraying drones are large-sized drones that can lift a heavy load because they carry several liters of liquid and spraying system components. In this study, a drone that can handle approximately 201t of liquid was designed. Therefore, the chassis must be strong, durable, and light. Aluminum was used in chassis construction because it is durable, cheap, light, and easily available. Drones are multi-rotor unmanned aerial vehicles. Generally, drones with 4,6 , or 8 rotors are available. The number of rotors changes according to the weight of the load to be lifted or according to the motor-propeller pair used. It has been calculated that a 6 rotor drone can handle the designed structure when using a predetermined motor-propeller pair according to price and performance. The designed body consists of only 6 aluminum rods. 6 aluminum rods are uniquely assembled to create a large area in the middle where parts of the drone and spray system can be placed.

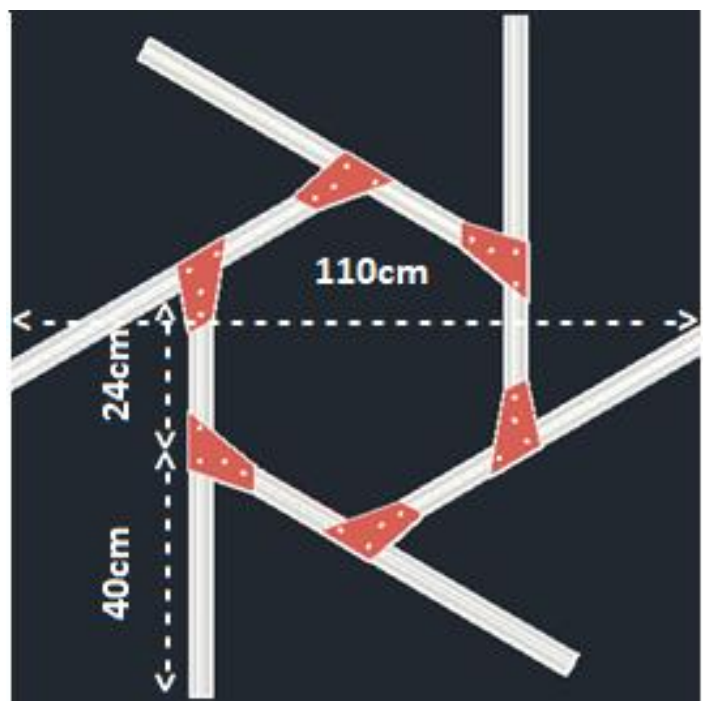

Figure 2. CAD drawing of the unique chassis.

The drone chassis shown in Figure 2 is of a unique structure and is very simple, robust, and reliable. By changing the connection points of the 6 rods to each other, the size of the area in the center can be adjusted easily. The rods are fixed to each other so that the 201t liquid drum can be easily mounted in the center of the drone. The distance between two opposing rotors is $110 \mathrm{~cm}$. The drone dimensions are shown in Figure 2.

\subsection{Wiring Diagram and Controller}

The drone has 6 rotors (hexacopter structure) and its electrical connection diagram is given in Figure 3. As seen in Figure 3, brushless motors are used in the drone. Electronic speed controllers (ESC) are used to control brushless motors, and the PWM signal required for ESCs is sent by the microprocessor through the flight software. 


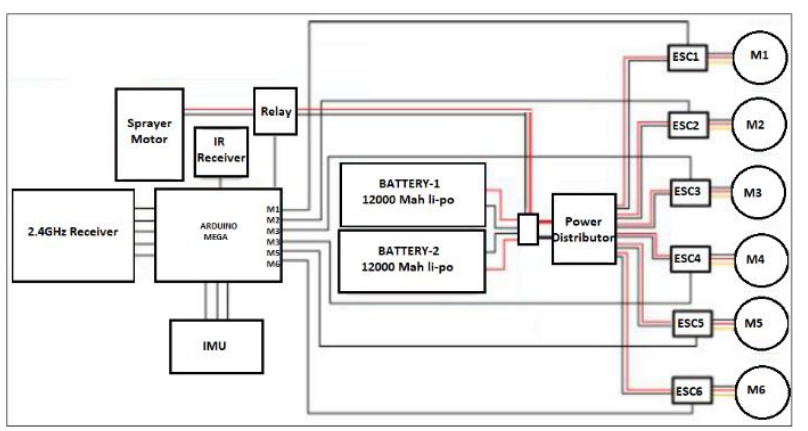

Figure 3. Electrical wiring diagram of the drone.

Flight software is not commercial software, it is original software developed by our team. Therefore, in case of any additions or changes, it can be easily overcome. Thus, a flexible working environment is provided. Thanks to the electrical power distributor, the necessary electrical power is given to the ESCs and motors. For spraying, a motor and a relay for turning the motor on and off were used. Commands from the IoT system are transmitted to the drone control system via an infrared receiver. In addition to being able to control the drone over the internet, it can also be controlled with a shortrange remote control. For this, a $2.4 \mathrm{GHz}$ radiofrequency $(\mathrm{RF})$ receiver is integrated into the system.

The wiring diagram has been introduced, and now information about drone control and controller design will be given.

Drones are vehicles that move in $\mathrm{x}, \mathrm{y}$, and $\mathrm{z}$ axes. Control is based on controlling the angle the drone chassis has to do with the axes. As in Figure 4, it is thought that the center of the drone is placed in the origin, the front-rear direction of the drone is placed in the $\mathrm{x}$-axis direction, the right-left direction of the drone is placed in the y axis direction. The angle between the front-rear direction of the drone and the $\mathrm{x}$-axis is called pitch angle, the angle between the right-left direction of the drone and y-axis is called roll angle and the angle of the drone center with the origin in the $\mathrm{z}$-axis is called the yaw angle.

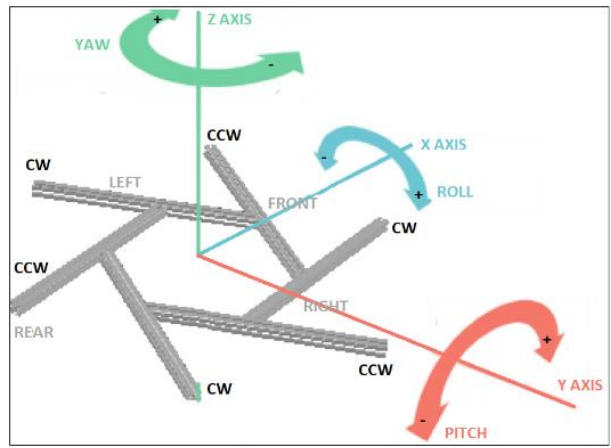

Figure 4. Yaw, pitch, roll angles, and motor rotation directions.
Yaw, pitch, and roll angles are controlled by rotating the drone rotors at different speeds. For example, if the motors on the left are rotated faster than the motors on the right, the drone moves to the right by making a roll angle in the + direction. Likewise, if the motors in the front are rotated faster than the rear ones, the drone makes a pitch attitude in the - direction and moves back. Or if the clockwise rotating motors are rotated faster than the counter-clockwise rotating motors, the drone would turn in the - yaw direction and turn counterclockwise.

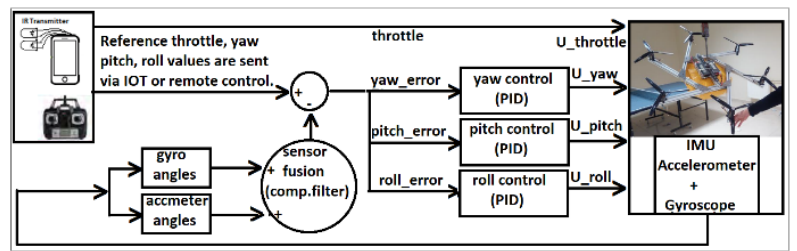

Figure 5. Control system flow chart.

Figure 5 shows the control system flow diagram. PID controller is used as the controller. The PID controller tries to eliminate the error by comparing the reference input values with the current values of the system. PID works in the same way on the drone. After the reference yaw, pitch, and roll angles are sent by the user, PID works to eliminate the error by comparing reference angles with the current angles of the drone measured by the inertial measurement system (IMU). PID controller performs these operations mathematically with the following equation.

$$
u(t)=K_{p} e+K_{i} \int_{0}^{t} e d t+K_{d} \frac{d}{d t} e
$$

PID stands for proportional, integral, and derivative. Here, the error (e) is multiplied by the $\mathrm{Kp}$ coefficient and approached to the reference. The error is eliminated over time by taking the integral of the error and multiplying it by the Ki coefficient. The sudden changes of the error are absorbed by taking the derivative of the error and multiplying it by the Kd coefficient. Many systems can be controlled with the PID controller. However, the $\mathrm{Kp}, \mathrm{Ki}$, and $\mathrm{Kd}$ coefficients are different for each different system. It is important to adjust these coefficients correctly, because the more accurate the adjustment is, the better the design of the controller. There are many different combinations of $\mathrm{Kp}, \mathrm{Ki}$, and $\mathrm{Kd}$ values for flight. However, one of them is the most ideal one. Adjusting means finding the most ideal coefficients, or those closest to it. Although these parameters are calculated by methods such as the Ziegler-Nichols method, it is more effective to adjust by experimenting in practice. While adjusting by experimenting, random values are determined first, then the coefficients are increased or decreased according to system response. While adjusting, it should be noted that $\mathrm{Kp}$ is responsible for the system speed, $\mathrm{Ki}$ for error elimination, and $\mathrm{Kd}$ for eliminating fluctuations in the 
system response. It is very difficult to adjust these coefficients experimentally in some systems, especially in non-stationary moving systems. Drones are one of these systems because of their three-dimensional movement. Because the coefficients should be in the range of coefficients required for a successful flight. If coefficients are chosen outside of this range, the drone will crash and be damaged so it will damage the environment and living things. Thus, drone production will be very costly and dangerous. To avoid such disadvantages, an adjustment should be made on a test platform. On the test platform, the drone is free as if flying in the air, but it cannot change position. In other words, it performs yaw, pitch, and roll movements, but the changing position is prevented. Within the scope of the study, a unique test platform has been developed as shown in figure 6.

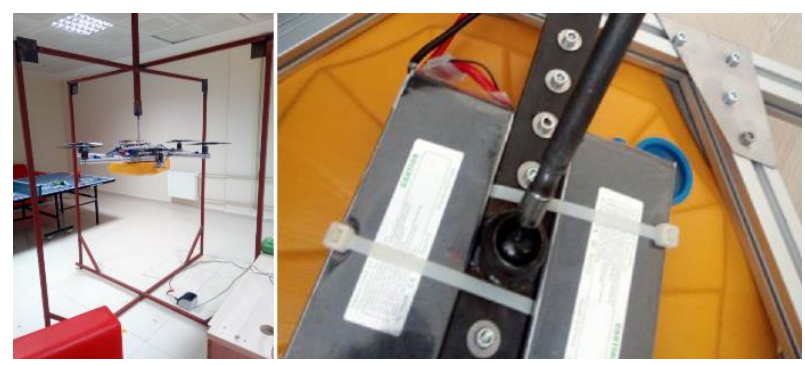

Figure 6. Drone test platform.

The test platform is designed in such a way that the drone can make its yaw, pitch, and roll movements without changing its position. To do this, the drone is attached to the flexible joint from the center. The flexible joint used is the car joint rod shaft. Thanks to the test platform, the controller parameters can be adjusted easily and the drone has been made to fly without damaging anything.

The last thing to mention is the IMU. With IMU, the instantaneous yaw, pitch, and roll angles of the drone are fed back to the controller as shown in Figure 5. The controller controls the drone from this information that comes with feedback. The IMU includes a gyroscope and an accelerometer to calculate the angles. The accelerometer measures linear accelerations, while the gyroscope measures angular acceleration. The data of the two sensors are combined with sensor fusion algorithms to obtain the drone's yaw, pitch, and roll angles. Many sensor fusion algorithms are available. Some of those; Kalman Filter, first-order complementary filter, second-order complementary filter, and yaw compensated complementary filter [19]. Although these sensor fusion algorithms do the same job, they differ in speed and immunity to noise. In the tests performed, it was observed that the most successful one of these algorithms for the designed drone is the yaw compensated complementary filter. Mathematically, how angles are obtained by combining raw sensor data is presented with the following equations. The calculations in the following equations are made for roll angle only, calculations for other angles are similar.

Gyro rate to gyro angle integration;

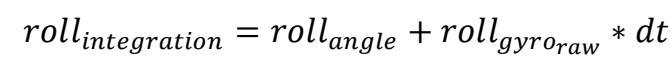

Yaw rate integration to roll angle;

$$
\begin{gathered}
\int \text { roll }_{\text {integration }}+\text { roll }_{\text {angle }} * \frac{\pi}{180} * \text { yaw }_{\text {gyro }} \text { raw } \\
* d t
\end{gathered}
$$

Error correction with roll accelerometer data

$$
\begin{gathered}
\text { roll }_{\text {error }}=\text { roll }_{\text {accel }}-\text { roll }_{\text {integration }} \\
\text { roll }_{\text {angle }}=\text { roll }_{\text {integration }}+\text { time }_{\text {cons }} \\
* \text { roll }_{\text {error }}
\end{gathered}
$$

Here the time constant is chosen between 0 and 1 .

\subsection{IoT System}

One of the most important devices of the IoT revolution is smartphones that have become a part of our lives. For this reason in this study, IoT application was carried out using an onboard smartphone. The implemented system is presented in Figure 7 and is unique.

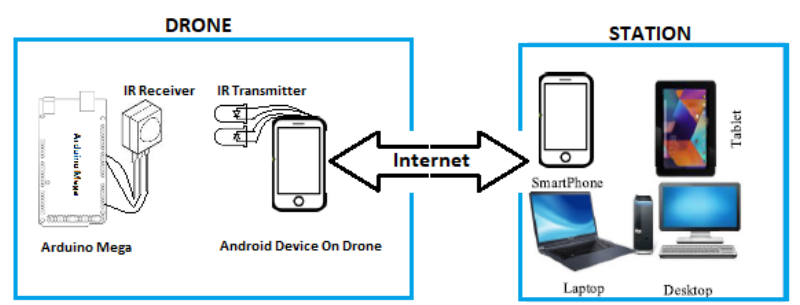

Figure 7. The implemented IoT system.

As seen in Figure 7, there is a smartphone on the drone. When the user wants to control the drone, he/she accesses the smartphone on the drone via another smartphone, tablet computer, or PC that can connect to the internet. This inter-device access is realized with the remote desktop application. Thanks to remote desktop access, it is possible to use the phone on the drone and send commands to the drone using it. It is not possible to do this directly. It is also necessary to establish a connection between the phone on the drone and the microprocessor containing the drone flight software. This connection can be made by wi-fi or Bluetooth. For this, it is necessary to attach a wi-fi or Bluetooth module to the microprocessor. It is possible to see many studies or applications in the literature that make connections in this way. This causes extra costs and can lead to negativity due to disconnection problems. In this case, the unmanned aerial vehicle will travel freely or even crash. A unique system has been proposed to overcome these problems. Wi-fi or Bluetooth is not used in the proposed system. Therefore, there is no problem of connection or disconnection. The proposed system is the same as the communication between television and remote control. This communication is infrared (IR) 
communication. The receiver is always ready to receive data from the transmitter. As soon as data is sent from the transmitter, the receiver starts to receive the data, and communication is established. This system is very useful, reliable, cheap, and simple. Its cost is also very low because it consists of one IR receiver LED and two IR transmitter LEDs. In this system, the encoded audio file played from the smartphone is converted to infrared waves. In other words, the smartphone gains infrared remote controller features like a TV remote controller. One IR receiver is attached to the microprocessor on the drone. Two IR transmitter LEDs are used in the circuit that converts the audio data from the smartphone's headphone output to IR signal, as in Figure 8.

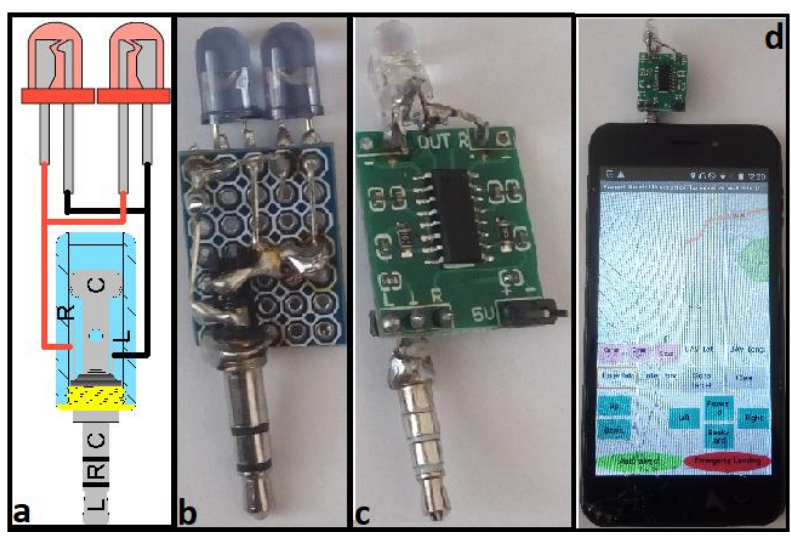

Figure 8. The circuit that converts encoded sound waves to IR waves.

The circuit that converts the sound to the IR signal is simple and it has two types. The first circuit is the circuit given in figure 8.b and is the same as the circuit given in figure 8.a. This circuit is for the sound card electrically powerful. It can be used by plugging it directly into the headphone jack. The second circuit is given in figure 8.c. The difference between this circuit and the other circuit is that it is used with a mini audio amplifier. It is necessary to apply external 5V DC to this circuit. From the moment the circuit is attached to the smartphone, it is possible to manage the device that works with an IR remote controller. Figure 8.d shows the circuit connected to the headphone output of the smartphone. In other words, the smartphone has become a remote control that manages many electronic devices such as televisions, air conditioners, and stereos. All that needs to be done is to convert the different IR signals emitted by the original remote controller keys into an audio file. Because the designed circuit is a circuit that converts audio signals into IR signals. How this conversion process is done can be learned in detail from this reference[20].

\subsection{Smartphone User Interface Application Design}

A useful user interface design has been developed within the framework of the study. The application is an android based application. The android platform was chosen because it is widely used, easy to develop, and cheap. The application is installed on the android smartphone on the drone. This application sends encoded audio to the device that converts the audio to IR. It is also an application that easily accesses the hardware such as the camera and GPS of the android phone it is installed on. The user uses this application by accessing the smartphone on the drone with a remote desktop connection from a computer, tablet, or other smartphones. Many remote desktop applications are available. TeamViewer application was used in the experiments. It can be downloaded for free on the Android market. Thanks to the remote desktop software, another paired device can be accessed and managed. In this study, the drone is also managed with the user interface developed by accessing the smartphone on the drone via the remote desktop application wherever you are in the world.

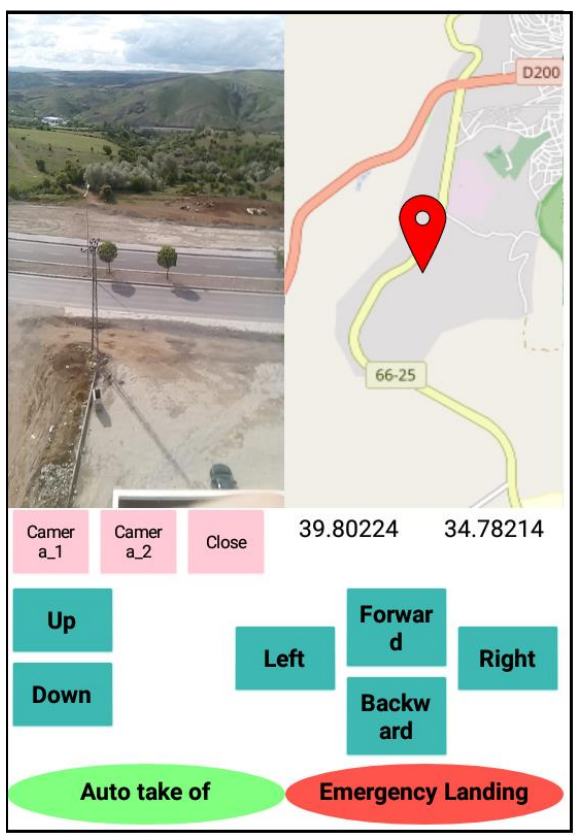

Figure 9. Android-based user interface application.

Figure 9 shows the screenshot of the designed user interface. To mention briefly; the Camera_1 button opens the rear camera of the smartphone on the drone, this camera shows the earth while the drone is air. The Camera_2 button opens the front camera of the phone, this camera shows the sky when the drone is in the air. The camera view is instantly seen in the upper left half of the user interface. The camera view is closed with the Close button. In the upper right half of the user interface, the Google Maps application instantly shows the position of the drone on the map, the coordinate information is also shown just below. For this, it is necessary to have a GPS sensor in the onboard android phone. With the control buttons on the lower half of the user interface, the drone is moved in the directions written on the buttons. These buttons are assigned differently coded sound waves. Pressing the button 
plays a short sound from the phone's music player. The played sound is converted into IR waves with the device connected to the headphone output and a command is sent to the drone. Since the response of the command generated by each key is pre-coded into the drone flight software, the drone knows which button is pressed and acts accordingly.

\subsection{Spraying system}

The spraying system consists of one yellow-colored 20 lt water container mounted under the drone (shown in Figure 6), one small water motor in the container, and six sprinklers. The durable water container, which has a large surface area, is also used as a stand.

While designing the spraying drone, elements with a high price/performance ratio were preferred. The most important of these elements are electric motors. As a result of the market research, $380 \mathrm{kV}$ motors with a power of $2665 \mathrm{~W}$, operating with $22.4 \mathrm{~V}$, which have a higher price-performance ratio than their competitors, were preferred. The thrust of these electric motors determined the other elements of the aircraft and the load it could handle. The thrust force of the selected electric motor and propeller pairs was calculated with the following formula.

Here;

$$
T=\left[(\eta x P)^{2} x 2 \pi x r^{2} \rho\right]^{1 / 3}
$$

$\eta$ : propeller efficiency (between 0.7-0.9), preferred:0.8

$P$ : electric motor power (Watt), preferred:2665 Watt

$r$ : radius of propellers $(\mathrm{m})$, preferred: $0.203 \mathrm{~m}$

$\rho$ : air density $\left(1.22 \mathrm{~kg} / \mathrm{m}^{3}\right)$

Calculated thrust is $=112.2 \mathrm{~N}$,

$1 \mathrm{~N}=0.101972 \mathrm{kgf}$

Then thrust is $=11.421 \mathrm{kgf}$ for one electric motor.

The UAV has 6 motors, so total thrust generated by all motors is $6 \times 11.421=68.526 \mathrm{~kg}$.

This means that the preferred electric motors and propellers can handle a total load of $68,526 \mathrm{~kg}$. However, for a successful flight, electric motors need to provide 1.5-2 times the thrust of the total weight of the aircraft. According to this calculation, the total weight of the aircraft should be at most $68.526 / 2=34.263 \mathrm{~kg}$. Since the curb weight of the aircraft is $16,161 \mathrm{~kg}$, it can carry $34.263-16.161=18.102 \mathrm{~kg}=18.102 \mathrm{lt}$ (water) cargo. Of course, this is the case when the factor of safety 2 is selected. If the safety factor is chosen between 1.5 and 2, there will be a carrying capacity of around 20 liters, so a 20lt tank has been selected.

For loads up to $20 \mathrm{lt}$, the desired amount of pesticide can be placed in the container and spraying can be done without making any changes. It is not suitable for loads more than 20 liters due to the maximum carrying limit of the aircraft.
Since the spraying process is not done autonomously, the success of the spraying depends on the user experience and the characteristics of the pesticide. The pesticide will be sprayed by the user at the optimum level.

\subsection{Cost of the System}

The cost of the total system is given in Table 1 .

Table 1. Cost of the system.

\begin{tabular}{|c|c|c|c|c|}
\hline & Product & Qty & Unit(\$) & Total (\$) \\
\hline 1 & 201t Container & 1 & 4,1 & 4,1 \\
\hline 2 & $\begin{array}{l}\text { Aluminium sigma rod } \\
35 \times 35\end{array}$ & $6 \mathrm{mt}$ & 65 & 65 \\
\hline 3 & Connection parts & 1 & 12 & 12 \\
\hline 4 & $\begin{array}{l}22.2 \mathrm{~V} \mathrm{Li}-\mathrm{Po} \text { Battery } \\
12000 \mathrm{mAh} 25 \mathrm{C}-6 \mathrm{~s}\end{array}$ & 2 & 282 & 564 \\
\hline 5 & Atmel microprocessor & 1 & 30 & 30 \\
\hline 6 & 9 Axis IMU & 1 & 37 & 37 \\
\hline 7 & 380KV Brushless Motor & 6 & 62,89 & 377,34 \\
\hline 8 & 200A ESC & 6 & 36,11 & 216,66 \\
\hline 9 & 3-blade Propeller 16x10 & 6 & 18,98 & 113,88 \\
\hline 10 & IR transmitter LEDs & 2 & 0,25 & 0,5 \\
\hline 11 & IR receiver & 1 & 0,5 & 0,5 \\
\hline 12 & $\begin{array}{l}\text { PAM8403 mini Audio } \\
\text { amplifier }\end{array}$ & 1 & 1 & 1 \\
\hline 13 & $\begin{array}{l}2.4 \mathrm{GHz} \text { Remote } \\
\text { Controller }\end{array}$ & 1 & 59,5 & 59,5 \\
\hline 14 & Water pump & 1 & 10 & 10 \\
\hline 15 & Li-po charger & 1 & 58 & 58 \\
\hline 16 & Old android smartphone & 1 & --- & --- \\
\hline \multicolumn{4}{|c|}{ TOTAL } & $1549,48 \$$ \\
\hline
\end{tabular}

\section{Results and Discussion}

As a result, a drone with different features than existing drones in the literature was designed and successfully flown.

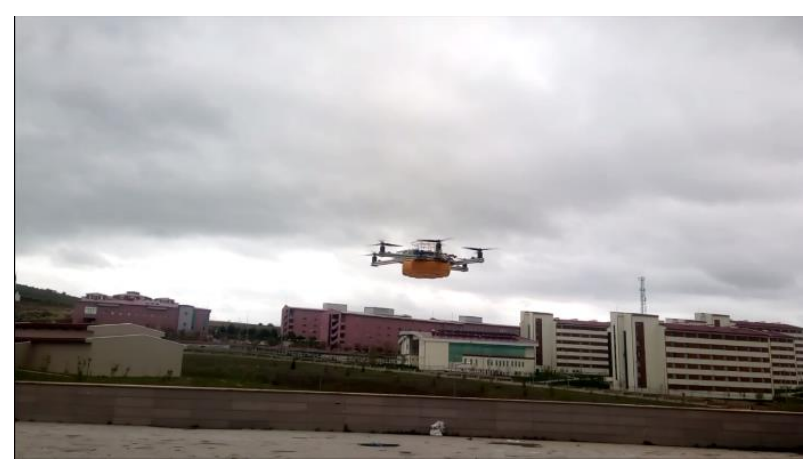

Figure 10. An image from the test flight. 
Especially the chassis design and IOT system are different and unique from existing drones. The center of the chassis is designed to expand and contract easily according to the water container to be mounted. Thus, for a heavy lift drone, there is easily place for all the elements. Using an onboard android system for the IoT system and controlling it with a remote desktop application has provided a lot of flexibility and convenience. The most important of these facilities is that the applications available in the android market and used for agricultural purposes are easily and instantly installed and the plant or field is analyzed and intervened quickly. In addition, a user with an android phone can have an IoT system at no extra cost. Thanks to the recommended plug-and-use audio-infrared converter, the user will be able to control drones from anywhere in the world. Thanks to the android smartphone, the user will not spend money on expensive equipment such as a camera, GPS, modem. In the IoT system, the communication between the internet provider device and the microprocessor responsible for flight is unique in that it eliminates connection problems and communication problems. Thanks to the developed original flight software and test platform, all-purpose development and updates can be made.

\subsection{Comparison With Other Agricultural UAVs}

Compared to other prominent agricultural drone manufacturers, the prototype sprayer drone reveals that it is the most economical UAV in its class with a 20 liter payload and IOT technology.

\begin{tabular}{|c|c|c|c|c|}
\hline & Manufacturer & IOT & Capacity & Price (\$) \\
\hline 1 & Our UAV & $\begin{array}{l}\text { Directly with } \\
\text { UAV }\end{array}$ & 201 & $1549.48 \$$ \\
\hline 2 & DJI Agras T20 & $\begin{array}{l}\text { Via Remote } \\
\text { controller }\end{array}$ & 201 & $16511.9 \$$ \\
\hline 3 & Xaircraft P20 & Via Pilot Phone & 101 & $21500 \$$ \\
\hline 4 & RPA Pelicano & $\begin{array}{l}\text { Via Remote } \\
\text { controller }\end{array}$ & 161 & No information \\
\hline 5 & SHIYANLI & $\begin{array}{l}\text { Via Remote } \\
\text { controller }\end{array}$ & 201 & $19054 \$$ \\
\hline 6 & IHA TR 5 & $\begin{array}{l}\text { Via Remote } \\
\text { controller }\end{array}$ & 201 & $21186 \$$ \\
\hline
\end{tabular}

Comparison made in terms of IoT, tank capacity and price. Except for these drones, drones with lower specifications are not included in the comparison. Fixed-wing unmanned aerial vehicles and helicopters are also not included in the comparison. The compared drones consist of drones that dominate the market with similar features to the drone we have designed. Our drone is an affordable drone compared to other drones. As can be seen, all drones can be connected to the internet and controlled via their remote controllers. In other words, there is an intermediary remote controller between the internet and the drone. Although these drones are controlled over the internet, they cannot go out of the remote controller range. In other words, this system works when the remote controller is close to the drone. But in our design, the drone is directly connected to the internet and there is no need for an intermediary remote controller. It doesn't matter if the remote controller is near or far. In addition to the price advantage, the advantage of direct internet connection makes our drone superior.

\section{Conclusion}

In a conclusion, a product has been designed to lead the fusion of technology and agriculture in recent years, when agriculture 4.0 has been spoken in the world with industry 4.0. An innovation that makes agriculture easy and attractive beyond traditional farming methods has been introduced. Thanks to this innovative technology, time and labor are saved. Spraying in agriculture is one of the most important and difficult jobs. The spraying process, which is done by diluting with tons of water with traditional spraying methods, has been ended and it has been possible to spray without wasting water. Thus, waste of water is prevented. In the traditional method, due to the difficulty of spraying, the whole field was sprayed, but thanks to the proposed product, regional spraying was provided instead of the whole. Thus, the environment was protected and expenses were reduced by preventing excessive use of the drug. The proposed system allows comfortable spraying, especially in difficult, rugged, and hilly terrain. Due to the ease of spraying, there is an increase in yield. In addition to these, the difficulty of going to the field for spraying and other works has been eliminated, and it has become possible to obtain information about the field and spraying without leaving the house. Since the product makes it easier to do agricultural work remotely via the internet, agriculture is continued even if there are migrations to big cities.

Novelty of this study is the design of a spraying drone that allows the use of technology in agriculture with affordable price and superior features. Since the designed drone has direct IoT technology, it provides superiority to drones managed over the internet via remote control. Its high carrying capacity of 20 liters is higher than the carrying capacity of many drones. Another original aspect is that it offers sustainable and budget-friendly solutions for precision agriculture. Sending commands to the drone with remote desktop connection through the android interface developed by us by converting the audio signal into infrared signals is unique.

\section{Acknowledgement}

This study was supported by Yozgat Bozok University with the BAP project number 6602b-MMF/18-194. 


\section{Author's Contributions}

Cemil ALTIN: Drafted and wrote the manuscript, performed the experiment and result analysis.

Hasan ULUTAȘ: Performed material purchase and assembly works.

Eyyüp ORHAN: Performed the chassis design and CAD drawing.

Orhan ER: Supervised the study's progress.

Volkan AKDOĞAN: Assisted in some works.

\section{Ethics}

There are no ethical issues after the publication of this manuscript.

\section{References}

1. S. K. Mohapatra, J. N. Bhuyan, P. Asundi, and A. Singh 2016."A Solution Framework For Managing Internet Of Things (Iot)," The International Journal of Computer Networks \& Communications.; 8(6):73-87 doi 10.5121/ijcnc.2016.8606.

2. "World Employment and Social Outlook: Which sector will create the most jobs?" https://www.ilo.org/global/about-theilo/multimedia/maps-and-charts/WCMS_337082/lang-en/index.htm (accessed Jul. 22, 2020).

3. R. Vidhya and K. Valarmathi, 2018."Survey on Automatic Monitoring of Hydroponics Farms Using IoT," in Proceedings of the 3rd International Conference on Communication and Electronics Systems ICCES 2018; 2018: 125-128.doi: 10.1109/CESYS.2018.8724103.

4. B. Basnet and J. Bang, 2018."The State-of-the-Art of Knowledge-Intensive Agriculture: A Review on Applied Sensing Systems and Data Analytics," Journal of Sensors; 2018: 1-13. doi: 10.1155/2018/3528296.

5. K. W. Jaggard, A. Qi, and E. S. Ober, 2010."Possible changes to arable crop yields by 2050," Philosophical Transactions of the Royal Society B: Biological Sciences; 365(1554): 2835-2851. doi: 10.1098/rstb.2010.0153.

6. S. Chakraborty and A. C. Newton, 2011."Climate change, plant diseases and food security: An overview," Plant Pathology; 60(1): 2-14. doi: 10.1111/j.13653059.2010.02411.x

7. M. A. Jubair, S. Hossain, M. A. Al Masud, K. M. Hasan, S H. S. Newaz, and M. S. Ahsan, 2018."Design and development of an autonomous agricultural drone for sowing seeds," IET Conference Publications; 2018 (CP750): 6-9. doi: 10.1049/cp.2018.1598.

8. D. Gao, Q. Sun, B. Hu, and S. Zhang, 2020.“A framework for agricultural pest and disease monitoring based on internet-of-things and unmanned aerial vehicles," Sensors (Switzerland); 20(5): 1487. doi: 10.3390/s20051487.

9. M. V. Suhas, S. Tejas, S. Yaji, and S. Salvi, 2018.“AgrOne: An Agricultural Drone using Internet of Things, Data Analytics and Cloud Computing Features," 2018 4th International Conference for Convergence in Technology. I2CT 2018; 2018: $1-6 . \quad$ doi: 10.1109/I2CT42659.2018.9057995.

10. M. Romero, Y. Luo, B. Su, and S. Fuentes, "Vineyard water status estimation using multispectral imagery from an UAV platform and machine learning algorithms for irrigation scheduling management," Computers and Electronics in Agriculture; $\quad 147$ : 109-117. doi: 10.1016/j.compag.2018.02.013.

11. M. Reinecke and T. Prinsloo, 2017.“The influence of drone monitoring on crop health and harvest size," 2017 1st International Conference on Next Geneation. Computing Appications NextComp 2017; 2017: 5-10. doi: 10.1109/NEXTCOMP.2017.8016168.

12. L. G. Santesteban, S. F. Di Gennaro, A. Herrero-Langreo, C Miranda, J. B. Royo, and A. Matese, 2017."High-resolution UAV-based thermal imaging to estimate the instantaneous and seasonal variability of plant water status within a vineyard," Agricultural Water Management.;183:49-59, doi: 10.1016/j.agwat.2016.08.026.

13. B. Allred, N. Eash, R. Freeland, L. Martinez, and D. B. Wishart, 2018."Effective and efficient agricultural drainage pipe mapping with UAS thermal infrared imagery: A case study," Agricultural Water Management..; 197:132-137, doi: 10.1016/j.agwat.2017.11.011.

14. I. Wahab, O. Hall, and M. Jirström, 2018. "Remote Sensing of Yields: Application of UAV Imagery-Derived NDVI for Estimating Maize Vigor and Yields in Complex Farming Systems in Sub-Saharan Africa," Drones; 2(3): 28. doi: $10.3390 /$ drones 2030028

15. J. Huuskonen and T. Oksanen, 2018."Soil sampling with drones and augmented reality in precision agriculture," Computers and Electronics in Agriculture.; 154: 25-35. doi: 10.1016/j.compag.2018.08.039.

16. S. Spoorthi, B. Shadaksharappa, S. Suraj, and V. K. Manasa, "Freyr drone: Pesticide/fertilizers spraying drone - An agricultural approach, 2017." in Proceedings of the 2017 2nd International Conference on Computing and Communications Technologies, ICCCT 2017; 2017: 252255. doi: 10.1109/ICCCT2.2017.7972289.

17. C. KOÇ, 2017.“Tarımda Pestisit Uygulama Amacıyla Ekonomik Bir Drone Tasarımı ve İmalat1," Journal of. Agricultural Faculty Gaziosmanpasa Univ.; 34(2017-1): 94103. doi: $10.13002 /$ jafag4274.

18.

B. Dai, Y. He, F. Gu, L. Yang, J. Han, and W. Xu, "A vision-based autonomous aerial spray system for precision agriculture, 2017." in 2017 IEEE International Conference on Robotics and Biomimetics; 2018:1-7, doi: 10.1109/ROBIO.2017.8324467

19. "Shane Colton: Fun with the Complementary Filter / MultiWii." http://scolton.blogspot.com/2012/09/fun-withcomplementary-filter-multiwii.html (accessed Aug. 15, 2020).

20. "Remote control - 2: Sample your remote | Jumping Jack Flashweblog."

https://jumpjack.wordpress.com/2008/05/22/remote-control2/ (accessed Aug. 16, 2020). 